\title{
Zur Geschichte der Ultraschalldiagnostik - Entwicklung des Medizinischen Ultraschalls aus deutscher Sicht
}

Hrsgb.: B. Frentzel-Beyme, C. Jakobeit, H. Lutz, D. Nürnberg, M. Salaschek mit Beiträgen von 24 Koautoren Herausgabe durch Ultraschallmuseum e. V., Lennep, Eigenverlag, 2020

ISBN 978-3-00-064418-4

267 Seiten, > 300 Farb- u. SW-Bilder, Tabellen, Grafiken und Faksimileabb., Preis: $30 €$

Mit viel Arbeit und großem Engagement haben die Herausgeber in rund 20 Kapiteln den Weg des diagnostischen Ultraschalls in Deutschland dargestellt. Ein sehr schwieriges Unterfangen, denn wer war in den 1960er- bis 1980er-Jahren schon dabei und kann darüber authentisch etwa 50 Jahre später berichten? Das Entstehen dieses Werkes ist den Aktiven des Ultraschallmuseums zu verdanken, die den größten Anteil der Autorenschaft übernahmen. Die Autoren aus der frühen Zeit der Ultraschallanwendung verleihen dem Werk einen besonderen Reiz. Da zeigen sich die echten Pioniere, auch wenn es den Herausgebern nicht gelang, diesen Typus - wie sie in ihrem Vorwort schreiben - zu definieren.

Das Buch gliedert sich in 4 große Abschnitte, die sich in 20 Kapiteln unterteilen, jedem Kapitel ist ein Literaturverzeichnis angefügt. Nach der Kurzvorstellung des UItraschall-Museums folgt eine gehaltvolle Darstellung der Anfänge der Ultraschalldiagnostik bis 1960 (mit dem Schwerpunkt auf den angloamerikanischen Entwicklungen), danach die technischen Methoden der darauffolgenden Jahre unter Berücksichtigung der Ultraschallsicherheit.

Den größten Abschnitt nimmt die Entwicklung in den verschiedenen medizinischen Fachgebieten ein. Die einzelnen Kapitel sind formal und inhaltlich uneinheitlich. Meist werden, entsprechend dem Titel des Werkes, überwiegend die Entwicklungen in den frühen Jahren, in manchen Kapiteln jedoch bis zu aktuellen Entwicklungen zusammengefasst. Die Reihenfolge der Kapitel ist historisch, deshalb wird mit der Opthalmologie und Neurologie begonnen, die Kardiologie sticht mit einem Reabdruck eines Vortrags von Prof. S. Effert heraus. Erst danach kamen die Geburtshilfe/Gynäkologie, die Innere Medizin usw., und weit später die chirurgischen Fächer hinzu.

In den „weiteren Entwicklungen“, werden die interventionelle Sonografie, die Endosonografie, die Kontrastverstärker und die im Vergleich noch sehr junge Elastografie (It. Autor sei US und Palpation seit tausenden Jahren existent) vorgestellt. Abschließend werden die Ultraschallgesellschaften dargestellt. Neben DEGUM, EFSUMB und WFUMB wird entsprechend unserer 2-gleisigen Landesgeschichte die Entwicklung des Ultraschalls in der DDR recht umfassend und informativ zusammengefasst, eines der Highlights in diesem Werk, gerade für die im Westen aufgewachsenen Kollegen. Unerwähnt bleibt allerdings die großartige Reaktion unserer ÖGUM-Freunde, die ohne langes Diskutieren 1990 beim Dreiländertreffen in Bregenz die zahlreich angereisten Kollegen der formal noch existierenden DDR von den Kongressgebühren befreiten und die DEGUM ein eigenes „Begrüßungsgeld“ ausgab.

Auch fehlt, dass der DEGUM dank des klugen Zuwartens des damaligen DEGUM-Präsidenten Prof. van Kaick beim Zusammenschluss der beiden Gesellschaften z. B. der spätere Ausschluss unerwünschter Ehrenmitglieder erspart blieb. Die damalige DDR-Gesellschaft hatte sich bereits selbst "gereinigt" und von ihrem regimetreuen Vorsitzenden getrennt. Prof. Milner übergab unserem Präsidenten eine Mitgliederliste und das Vermögen der DDR-Gesellschaft (ca. 800 DM West). So wurde die DEGUM um viele aktive US-Begeisterte reicher, alle Mitglieder aus Deutschland West und Ost sahen darin eine gelungene Vereinigung zweier wissenschaftlicher Gesellschaften.

Die anhand der Kongressposter dargestellten Dreiländertreffen werden bei vielen älteren Kollegen nostalgische Erinnerungen an die frühen wissenschaftlich-innovativen und familiär-freundschaftlichen Kongresse hervorrufen. Neues erfuhr man damals noch im Kongresssaal und nicht aus dem Internet. Spätestens da vermisst man in diesem Buch als Mitglied der DEGUMGründer-Generation eine Darstellung der SGUM und ÖGUM - ohne unsere Schwestergesellschaften hätte sich das Dreiländertreffen so nicht entwickeln können.

Hinsichtlich des Untertitels „Die Entwicklung des Medizinischen Ultraschalls aus deutscher Sicht" fehlt die Vorgeschichte (vor 1945) mit den ausgiebigen Arbeiten zum anfangs nur „therapeutischen US“, heute wieder aktuell mit anderer Technik und Zielsetzung; auch kommen die technischen Entwicklungen in Deutschland West und Ost etwas zu kurz.

Sicherlich werden auch die Protagonisten der ersten „Ultraschallschulen“ der Bundesrepublik ihren Beitrag zu Ausbildung und wissenschaftlicher Verbreitung der Methode unterrepräsentiert sehen, ebenso wie ihren Beitrag in der Wendezeit. Da fällt die hervorgehobene Stellung eines regionalen US-Vereins auf, da gelingt der abwägende Blick auf die Geschichte des Ultraschalls in Deutschland nicht so recht.

Noch reichlich Interessantes hätte man aus den Kongressbänden der Dreiländertreffen herausfiltern können, denn es ist den jüngeren US-Anwendern nicht geläufig, dass nahezu alles, was die B-Bild-Technik bietet, bereits 1984 bekannt war. Da hätte ich mir eine schwerpunktmäßige Darstellung der wissenschaftlichen Aktivitäten gewünscht, zumal die Ultraschalldiagnostik damals hier, international gesehen, durchaus die Führungsrolle innehatte. Zu kurz kommt auch das Ultraschallmuseum, so fehlt z. B. der praktische Hinweis, wie man das Museum besuchen kann.

Was mir besonders fehlt ist ein Kapitel über die Erfolgsgeschichte der 1980 gegründeten „Ultraschall in der Medizin“, die sich zu einer der auflagenstärksten Ultraschallzeitschriften mit internationaler Wertschätzung entwickelt hat. Aber mit dem Buchtitel „Zur Geschichte der Ultraschalldiagnostik" und in 
ihrem Vorwort weisen die Herausgeber zu Recht daraufhin, dass Geschichte nie vollständig und abgeschlossen dargestellt werden kann.

Alles in allem ein interessantes Buch für alle Ultraschallinteressierten. Das sind zum einen die „Pioniere“, die anfänglich die Sonografie mitgestaltet, miterlebt und die Methode durchgesetzt haben, und andererseits die jüngeren Kollegen, die diese Zeit nun historisch „nacherleben“ können. Bei aller Kritik im Einzelnen: es gibt nichts Besseres. Das Werk lässt den mühsamen Weg zur klinischen Akzeptanz nachvollziehen, ebenso wie die kontinuierlichen methodischen „Fortschritte“; etwas mehr
Recherche und Sorgfalt hätte gleichwohl an manchen Stellen gutgetan. Das Buch belegt, wie sich die tägliche klinische Arbeit in nahezu 50 Jahren mit dem patientennahen Ultraschall bereichert hat.

Jungen „Ultraschallern“ zeigt das Werk zudem, wie viel einfacher die technische Anwendung und wieviel anschaulicher die Ultraschallbilder mittlerweile geworden sind.

Ein ideales Hilfsmittel ist das Buch sicherlich für Referenten - da findet jeder rasch einen historischen Einstieg für jedes Ultraschallthema. Das Werk sollte in keiner Ultraschallbibliothek fehlen, es ist reich illus- triert, für die Publikation im Selbstverlag besitzt es das übliche etwas „holprige“ Layout, doch als Hardcover mit Fadenbindung ist es nachhaltig gefertigt. Viele der oft kleinen Bilder sind von mäßiger Bildqualität, was größtenteils den „historischen“ Dokumentationsmöglichkeiten geschuldet ist.

Für $30 €$ pro Exemplar ist die Geschichte der Ultraschalldiagnostik außergewöhnlich preiswert.

Also sichern Sie sich ein Exemplar über bestellung@ultraschallmuseum.de.

\section{Karlheinz Seitz}

